\title{
FACHSPRACHLICHE KOLLOKATIONEN - SCHWERPUNKT DEUTSCH-SLOWENISCH
}

\section{EINLEITUNG}

„Fachsprache hat zwei Dimensionen, das Einzelwort, der Terminus, der meist im Zentrum der Terminologie steht, und die ebenfalls fachsprachliche Kontextualisierung des Terminus in seinen Kollokationen" (Hausmann 2003: 83). Die Suche nach äquivalenten Termini in der Zielsprache ist bei juristischen Texten nicht selten eine anspruchsvolle Aufgabe. Wer Rechtstexte übersetzt, hat es nämlich nicht nur mit einem Sachgebiet in zwei verschiedenen Sprachen zu tun, sondern mit zwei Rechtsordnungen in zwei unterschiedlichen Sprachen. Nicht nur die Auswahl entsprechender Termini stellt eine große Herausforderung dar, sondern auch die Auswahl entsprechender Verben, Adjektive und Präpositionen, die sich mit Termini verbinden, z. B.: einen Vertrag schließen/ aufheben $=$ skleniti/razveljaviti pogodbo, unter Verwendung einer Waffe $=z$ uporabo orožja, das Gesetz verabschieden = sprejeti zakon... Solche festen Wortverbindungen können selbst erfahrenen Übersetzern oft erhebliche Schwierigkeiten bereiten, weil sie eine Übersetzungseinheit bilden und daher nicht einzelsprachlich übersetzt werden können. Beim Fachtextübersetzen bereiten sie Schwierigkeiten nicht nur beim Hin-, sondern auch beim Herübersetzen. Der Grund dafür ist zum einen die Tatsache, dass jede Fachsprache meist Wortverbindungen enthält, die in der Gemeinsprache nicht zu finden sind; und zweitens: Viele dieser spezifischen Wortkombinationen sind in verschiedenen Nachschlagewerken (Glossaren, Lexika, Wörterbüchern...) nicht kodifiziert.

Der vorliegende Beitrag beschäftigt sich in erster Linie mit fachsprachlichen Kollokationen und mit der Notwendigkeit ihrer Aufnahme in Fachwörterbücher.

\section{ZUM BEGRIFF DER KOLLOKATION}

Der Begriff „Kollokation“ ist zunächst mit dem Namen des britischen Linguisten Firth - ,innerhalb der Schule des britischen Kontextualismus“ - verbunden, der damit „das faktische Miteinandervorkommen beliebiger Wörter“ bezeichnet (Lehr 1993: 2). Im Rahmen der deutschsprachigen Germanistik wird der Terminus meist eingeschränkt auf typische, usuelle, konventionelle und rekurrente Lexemverbindungen. So geht auch Hausmann (1985: 118) von ,wesenhaften Bedeutungsbeziehungen“ aus und unterscheidet zwischen wesentlichen und unwesentlichen Wortverbindungen. Nur die ersteren betrachtet er als Kollokationen, wobei das gleichzeitige Auftreten von zwei Elementen eine wichtige Voraussetzung ist. ${ }^{1}$ Er definiert die Kollokation als „typische, spezifische

Anders bei Firth (1957: 10), der das Phänomen als „coocurrence of lexical items“ definiert, womit keine notwendige Begrenzung des Umfangs der Kollokation vorgegeben ist. Demnach kann eine Kollokation aus zwei, aber auch aus mehreren lexikalischen Einheiten bestehen. Laut Jones/Sinclair (1973: 16, zitiert in Bergenholz/Tarp 1994: 404) kann auch ein ,gleichberechtigtes Miteinander-Auftreten“ von Lexemen 
und charakteristische Zweierkombination von Wörtern“ (ebd.), oder genauer: „die Kollokation ist die phraseologische Kombination von Basis und Kollokator" (Hausmann 2003: 83). Die Basis ist ein Wort, das ohne Kontext definiert, übersetzt und gelernt werden kann und determiniert den anderen Teil der Kollokation - den Kollokator, der nicht ohne Kontext definiert, übersetzt und gelernt werden kann (vgl. Hausmann 2003: 83), z. B.: ein Streichholz anreißen = anzünden. Während die Basis (Streichholz) keines Kontextes bedarf, ist das beim Kollokator (anreißen) nicht der Fall. Die Basen sind daher „semiotaktisch autonom", die Kollokatoren hingegen ,semiotaktisch abhängig“. Was die Wortart betrifft, so kommt als typische Basis ein Substantiv vor, zu dem sich dann als typischer Kollokator ein Adjektiv (ein starker Raucher, eine bittere Enttäuschung) oder ein Verb (ein Buch aufschlagen, die Begierde stillen) gesellt. Eine substantivische Basis kann aber auch einen substantivischen Kollokator besitzen: So hat Hitze als Kollokator Wallung $\rightarrow$ Hitzewallung, Zorn als Basis bildet eine Kollokation mit dem Kollokator Ausbruch $\rightarrow$ Zornausbruch (dagegen nicht ${ }^{*}$ Zornanfall, wohl aber Wutanfall, auch Wutausbruch) ${ }^{2}$ (vgl. Hausmann 2003: 85). Wie schon betont, betrachtet Hausmann als Kollokationen nur Kombinationen von zwei Wörtern, und bei diesen nur die, die in einem Determinationsverhältnis stehen. Wie aber aus den obigen Beispielen (Hitzewallung...) ersichtlich wird, zählt er zu Kollokationen nicht nur Wortverbindungen aus zwei getrennt geschriebenen Einheiten, sondern auch Komposita.

Ähnlich wie Hausmann macht auch Viehweger (1982) einen Unterschied zwischen banalen und typischen Wortkombinationen, z. B.: ein Buch kaufen (banal) / ein Buch aufschlagen (typisch), einen Vogel sehen (banal) / einen Vogel beobachten (typisch). Die Verbindung ein Buch kaufen sei im Gegensatz zu ein Buch aufschlagen untypisch, da die Aktion kaufen im Hinblick auf Buch banal sei. Bergenholtz/Tarp (1994: 406) halten das Relevanzkriterium für problematisch und führen dabei das Argument der Subjektivität ins Feld. Statt dessen schlagen sie eine frequenzorientierte Kollokationsselektion vor - die Selektion von auffälligen Kollokationen, die auch lexikographisch relevant sind.

An dieser Stelle soll noch eine wichtige Komponente der Kollokation angesprochen werden - nämlich ihre phraseologische Dimension. Burger (2003: 51) versteht unter dem Begriff „Kollokation“ jene festen Wortverbindungen, ,die nicht oder nur schwach idiomatisch sind“. Übrigens rechnet Burger (2003: 37 f.) Kollokationen dem Bereich der referentiellen Phraseologismen zu, die syntaktisch gesehen einem oder mehreren Satzgliedern entsprechen (nominative bzw. satzgliedwertige Phraseologismen). Nominative Phraseologismen lassen sich nach dem semantischen Kriterium der Idiomatizität des weiteren in drei Gruppen unterteilen: Idiome (idiomatische Wortverbindungen), Teil-Idiome (teil-idiomatische Wortverbindungen) und Kollokationen (nicht- bzw. schwach-idiomatische Phraseologismen).

Dieser phraseologische Akpekt der Kollokationen wird auch bei Hausmann (2003: 84) angesprochen; idiomatische Redewendungen kann man als Phraseme und die Kollokationen als Halbphraseme bezeichnen. Sowohl die einen wie auch die anderen sind im Gedächtnis der Sprecher als phraseologische Einheiten gespeichert. Der Unterschied

angenommen werden: „there ist no difference in status between node and collocate"; das heißt, jedes Element einer Kollokation kann als Basis (node) oder als Kollokator (collocate) gesehen werden.

2 Die slowenischen Entsprechungen: Hitzewallung = vročinski val, Zornausbruch = izbruh jeze, Wutanfall = napad/naval besa/gneva/popadel ga je bes/gnev. 
zwischen den beiden besteht aber darin, dass die Redewendungen keine Basis und nur en bloc Bedeutung haben - d.h. die verwendeten Wörter können nicht für sich selbst stehen, wie etwa jemanden durch den Kakao ziehen. Oder mit anderen Worten: Die Bedeutung dieser letzten Wortverbindung lässt sich nicht aus der Summe der Bedeutung der Konstituenten erschließen. Im obigen Beispiel geht es nicht um Kakao, während es sich in der vorher erwähnten Kollokation ein Streichholz anreißen sehr wohl um ein Streichholz handelt (vgl. Hausmann 2003: 84).

Fleischer (1997: 251 f.) rechnet Kollokationen zum nichtidiomatischen Typ der Phraseologismen. Da solche nichtidiomatischen Wortverbindungen nicht alle Kriterien der Phraseologie ${ }^{3}$ erfüllen, gehören sie nicht dem idiomatischen-teilidiomatischen Zentrum mit stabilen und lexikalisierten Wortverbindungen an, sondern der Peripherie, wo nichtidiomatische, aber mehr oder weniger feste Wortverbindungen zu finden sind. Nichtidiomatisch heißt bei Fleischer, die Bedeutung der Wortverbindung lässt sich aus der Summe der Bedeutungen der Konstituenten erschließen und ist somit kompositionell. Die Kompositionalität ist aber gerade bei Kollokationen strittig, u. z. wegen der semantischen Besonderheit des Kollokators. Die Bedeutung des Kollokators kann nämlich erst in Verbindung mit der Basis festgelegt werden (wie anreißen in ein Streichholz anreißen). Auffällig ist auch die Tatsache, dass die Verben oder Adjektive als Kollokatoren in den gegebenen Lexemverbindungen keine oder wenige Synonyme aufweisen, selbst wenn man rein theoretisch das gleiche mit anderen - synonymen - Verben bzw. Adjektiven ausdrücken könnte: *Kaffee nehmen (nur Kaffee trinken), *eingefleischter Raucher (wohl aber starker Raucher). In diesem Sinne sind auch Kollokationen als eine Bedeutungseinheit zu verstehen (vgl. dazu auch Caro Cedillo 2004: 38).

Einige Linguisten bezeichnen Kollokationen als idiomatisch, wobei die Idiomatizität nicht im Sinne der Undurchsichtigkeit der Bedeutung gemeint ist. So auch Hausmann (1995: 22): „Kollokationen sind durchsichtig, aber nicht vorhersehbar.“ Außerdem ist die Idiomatizität der Kollokation in erster Linie mit der Idiosynkrasie verbunden, mit der Eigenschaft „einzelsprachlich unterschiedlich“ (so z. B. das Bett machen = slow. postlati (posteljo), den Tisch decken = slow. pogrniti mizo = engl. to lay the table, Kaffee machen = slow. skuhati kavo, Salat putzen = slow. (na)trebiti solato, den Tisch abräumen = engl. to clear the table = slow. pospraviti z mize, unter die Dusche gehen/duschen = slow. oprhati se/iti pod prho = it. fare la doccia, sich die Zähne putzen = slow. umiti si $z o b e=$ fr. se laver les dents) aber auch - wie schon oben betont - mit der Spezifität des Kollokators, der keine oder sehr wenige Synonyme erlaubt. ${ }^{4}$

Während Idiome neben Idiomatizität auch das Kriterium der semantisch-syntaktischen Stabilität erfüllen, sind Kollokationen ,semantisch und syntaktisch regulär und weisen keine Besonderheiten auf" (Caro Cedillo 2004: 40). Sie sind syntaktisch flexibel und erlauben verschiedene Transformationen wie z. B. ein Streichholz anzünden, Streichhölzer anzünden, die kleinen Streichhölzer anzünden, das Streichholz wurde angezündet etc. Die Kollokationen weisen keine ,innere“, wohl aber eine „äußere Stabilität“ auf. Irsula

3 Die drei Hauptmerkmale der Phraseologie sind Idiomatizität, Stabilität und Lexikalisierung (vgl. Fleischer 1997, Kapitel 2. 1, 2. 2, 2. 3). Zu betonen ist jedoch in diesem Zusammenhang, dass sich Fleischers Untersuchungen auf die gemeinsprachliche und nicht auf die fachsprachliche Phraseologie beziehen.

4 Synonymie beim Kollokator: eine Rolle spielen = slow.: imeti vlogo, auch igrati vlogo - wobei das Letztere umgangssprachlich ist, weit verbreitet, schriftsprachlich jedoch nicht korrekt; im Recht: ein Testament errichten, in der Praxis statt errichten auch machen als Synonym weit verbreitet). 
(1994: 17) unterscheidet in Anlehnung an Coulmas (1981) zwischen einer inneren und einer äußeren Stabilität. Während sich die innere Stabilität auf die syntaktische und semantische Struktur der Wortverbindung bezieht, ist die äußere, pragmatische Stabilität ,auf die Rekurrenz des Miteinandervorkommens in bestimmten kommunikativen Situationen oder Textsorten, für den Ausdruck rekurrenter Sachverhalte reduziert" (Caro Cedillo 2004: 41).

\section{TERMINI UND KOLLOKATIONEN IN FACHWÖRTERBÜCHERN - ABGRENZUNGSPROBLEMATIK}

Beim Erstellen von Fachwörterbüchern oder terminologischen Datenbanken ergeben sich nicht selten Schwierigkeiten in Bezug auf die Abgrenzung der Kollokationen von den Mehrworttermini. Eine klare Trennung - vor allem nominaler Kollokationen (Adjektiv-Substantiv- oder Substantiv-Substantiv-Verbindung) und Mehrworttermini - ist trotz (zahlreicher) Definitionen der beiden Begriffe oft nicht einfach. „Mehrworttermini werden von den nominalen Kollokationen durch die Kriterien, dass sie eine neue ,gut definierte Realität‘ ausdrücken (Cohen 1993: 507 und Reichling 1993: 488), aufgrund ihrer Zugehörigkeit zur Fachsystematik (Bergenholtz/Tarp 1994: 401) sowie aufgrund ihrer begrifflichen Autonomie (Schneider 1998: 82) abgegrenzt" (Caro Cedillo 2004: 90). Auf der anderen Seite haben aber auch Kollokationen einen eigenen Referenten, drücken fachspezifische Sachverhalte aus, bilden eine semantische Einheit und lassen sich nur zusammen übersetzen (vgl. Caro Cedillo, ebd.).

Die Abgrenzung der Kollokationen gegenüber den Mehrworttermini ist für die fachsprachliche Lexikographie von großer Bedeutung; durch eine (klare) Systematik könnte man nämlich eine Belastung der Makro- und Mikrostruktur des Wörterbuchs vermeiden und dadurch den Benutzern einen leichteren Umgang mit dem Nachschlagewerk ermöglichen. Dies lässt sich anhand des folgenden Beispiels beweisen: Die im Recht oft vorkommenden Ausdrücke Vollmachtserteilung und Vollmachts-überschreitung werden in verschiedenen Nachschlagewerken ${ }^{5}$ sehr heterogen behandelt. Im deutschslowenischen Rechts- und Wirtschaftswörterbuch von Apovnik/Karničar (1989: 360) sind unter dem Lemma Vollmacht unter anderem auch die Kollokationen eine Vollmacht erteilen (= dati pooblastilo) und eine Vollmacht überschreiten (prekoračiti pooblastilo) angeführt. Außerdem finden sich in diesem Wörterbuch auch ihre nominalisierten Formen, u. z. als selbstständige Lemmata - Vollmachtserteilung (= pooblastitev) und Vollmachtsüberschreitung (= prekoračitev pooblastila). Im Rechtswörterbuch von Creifelds (1996: 1417-1419, ein umfangreiches einsprachiges Rechtswörterbuch, das Termini und ihre Definitionen, aber keine Kollokationen enthält) sind nur Vollmacht und Vollmachtmißbrauch lemmatisiert, dagegen nicht Vollmachtserteilung und Vollmachtsüberschreitung. Ähnlich auch im Rechtswörterbuch von Köbler (1998: 441), wo nur Vollmacht vorkommt. Im juristischen Lehrbuch zum bürgerlichen Recht von Koziol/

5 Die analysierten Nachschlagewerke: Apovnik/Karničar (1989): Deutsch-slowenisches Rechts- und Wirtschaftswörterbuch (zweisprachig, mit Übersetzungsäquivalenten und Kollokationen, Adressaten sind m. E. in erster Linie Übersetzer, d. h. Laien); Creifelds (1996): Rechtswörterbuch (einsprachig, Termini mit dazugehörigen Definitionen, für Juristen und Laien bestimmt); Köbler (1997): Juristisches Wörterbuch. Für Studium und Ausbildung (einsprachig, Termini mit Definitionen, der Adressatenkreis: vor allem die Studierenden im Fach Recht). Das Wörterbuch von Apovnik/Karničar ist das einzige für das Sprachenpaar Deutsch-Slowenisch, das auch Kollokationen - u. z. in den beiden Sprachen - enthält. 
Welser (1995) findet sich im Sachverzeichnis nur Vollmachtsüberschreitung, u. z. mit dem Verweis auf entsprechende Seiten im Buch. Im Text selbst wird der Ausdruck dann nicht definiert; es sind lediglich Fälle der Vollmachtsüberschreitung dargestellt, u. z. Vertretung ohne Vertretungsmacht, Mißbrauch der Vertretungsmacht und Insichgeschäft. Natürlich drängt sich hier folgende Frage auf: sind Vollmachtserteilung und Vollmachtsüberschreitung Termini, oder geht es dabei lediglich um die Nominalisierung der Kollokationen? Als Nicht-Fachmann/Fachfrau würde man sich in Anlehnung an die oben dargestellten Tatsachen eher für die letzte Möglichkeit einsetzen. Aus fachlicher Sicht ist die oben dargestellte Problematik jedoch anders zu bewerten: Vollmachtserteilung, die auch als Synonym für Bevollmächtigung verwendet wird, bezieht sich auf einen genau definierten Begriff und kann in diesem Sinne als Terminus aufgefasst werden. Auch bei der Vollmachtsüberschreitung ist das sehr wahrscheinlich ${ }^{6}$ der Fall, denn dabei handelt es sich nicht nur um die Konkretisierung bzw. Ergänzung des Terminus Vollmacht (eine Vollmacht überschreiten), sondern um den Übergang in einen neuen Zustand mit neuen Rechtsfolgen. Vollmachtsüberschreitung stellt inhaltlich eine selbstständige Einheit dar und ist demzufolge auch als Terminus einzustufen. Zum Vergleich kann man beispielsweise Notwehrüberschreitung (prekoračitev silobrana/prekoračeni silobran) nehmen, die als Terminus zählt. Merkwürdig ist dabei, dass der Ausdruck sowohl im deutschen Rechtswörterbuch von Köbler wie auch im deutsch-slowenischen Wörterbuch von Apovnik/Karničar vorkommt, nicht dagegen im juristischen Wörterbuch von Creifelds. Erwähnenswert ist in diesem Zusammenhang noch Testamenterrichtung, die im Wörterbuch von Apovnik/Karničar angeführt ist, u. z. sowohl in der Nominalform als Lemma, wie auch als Kollokation Testament errichten (unter der Basis Testament). Im Gegensatz zu Vollmachtserteilung und Vollmachtsüberschreitung gilt sie jedoch nicht als Terminus, sondern als Kollokation. Das genannte Wörterbuch verfährt gleich bei Termini und Kollokationen - alle sind lemmatisiert. Diese Methode ist meiner Meinung nach unsystematisch, da Termini und Nicht-Termini gleich behandelt werden; darüber hinaus ist sie aber auch unökonomisch, da sie viel Platz in Anspruch nimmt.

Es handelt sich hier um ein wesentliches Problemgebiet, das beim Konzipieren von Fachwörterbüchern unbedingt mehr Beachtung verdient. Lexikographen bzw. Linguisten verfügen nur ausnahmsweise über ein ausreichendes Fachwissen; dieses ist aber beim Erstellen eines Fachwörterbuches eine unerlässliche Voraussetzung, denn zwischen der allgemeinsprachlichen Lexikographie und der Fachlexikographie liegt ein radikaler Unterschied vor (vgl. Bergenholtz/Tarp 1994: 401). Eine von Fachleuten ausgearbeitete Fachsystematik, die als Grundlage für die fachlexikographische Arbeit dienen kann, schließt sowohl die Klassifizierung von Termini wie auch die Selektion der fachlich relevanten Kollokationen ein. In Anlehnung daran wären nur Termini lemmatisiert, Kollokationen kämen lediglich als Angaben im jeweiligen Wörterbuchartikel vor; dies ist jedoch nur eine von zahlreichen Möglichkeiten der makro- und mikrostrukturellen Gliederung des Fachwörterbuches (mehr hierzu vgl. Bergenholtz/Tarp 1994: 393 ff.). Wichtig ist in diesem Zusammenhang auch die Frage, wo die Kollokationen angegeben sind - unter

\footnotetext{
${ }^{6}$ Selbst die Fachleute können nicht immer mit Sicherheit sagen, ob es sich bei einer Wortgruppe/einem Kompositum um einen Terminus oder eine Kollokation handelt. „Nur ein großes Team von Fachleuten aus den vorgesehenen Fächern könnte entscheiden, ob es sich in den jeweiligen Fächern um Termini handelt oder nicht" (Bergenholtz/Tarp 1994: 392).
} 
der Basis oder unter dem Kollokator. Im Fachwörterbuch von Apovnik/Karničar zeigt sich diesbezüglich eine gewisse Unsystematik, u. z. viele Kollokationen kommen sowohl unter dem Kollokator wie auch unter der Basis vor, z. B.: verabschieden (=Kollokator): ... ein Gesetz verabschieden izglasovati zakon, sprejeti zakon; Gesetz (= Basis): ... ein Gesetz verabschieden sprejeti zakon. Diese Vorgehensweise ist platzverschwendend und belastet sowohl die Makro- wie auch die Mikrostruktur des Wörterbuchs. „Auf Kollokationsangaben spezialisierte Wörterbücher sollen sich auf Basisartikel beschränken und diese möglichst vollständig mit Kollokationen füllen. So helfen sie dem Benutzer wirksam beim Formulieren von Texten“ (Hausmann 2003: 87). Dieses Prinzip ist also vor allem für Produktionswörterbücher geeignet (vgl. auch Hausmann 1985), d. h. für die Wörterbücher, die den Übersetzern beiseite stehen sollen.

\section{BEDARF AN KOLLOKATIONSANGABEN}

„Eine wortwörtliche, an den Strukturen der Ausgangssprache orientierte Übersetzung, oder eine entsprechend der Strukturen der Zielsprache unübliche Übersetzung von Kollokationen führt notgedrungen zu Fehlübersetzungen [...]" (Caro Cedillo 2004: 32), z. B.: einen Unterbevollmächtigten bestellen = *narociti namestnika (richtig: postaviti/imenovati namestnika), eine Norm verletzen $=$ *poškodovati normo (richtig:kršiti

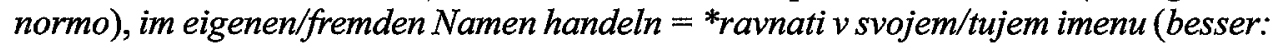
nastopati v...), Rechte/Freiheiten achten/schützen = *pravice/svoboščine upoštevati/ šcititi (richtig: spoštovati/varovati), in Kraft treten $=*$ priti v veljavo/pričeti veljati (besser: stopiti v veljavo)... Fachsprachliche Kollokationen bereiten Probleme nicht nur beim Hin-, sondern auch beim Herübersetzen. Die Übersetzer müssen die Kollokationen im Ausgangstext erkennen und dann in der Muttersprache typische Kollokationen wählen, z. B.: das Gesetz verabschieden = sprejeti zakon, zur Last legen = obtožiti/ vložiti obtožbo (zoper), die Mängel beheben = odpraviti napake, in Rechte eingreifen = posegati $v$ pravice... ${ }^{7}$ Diese und folgende Kollokationen sind in der Rechtssprache sehr gängig; da sie aber fachspezifisch sind, bereiten sie als solche den Übersetzern nicht selten (große) Schwierigkeiten. Die obigen Kollokationen kommen im Rechtswörterbuch von Apovnik/Karničar vor, wohingegen es für folgende entweder kein Äquivalent gibt, Informationen darüber in diesem Wörterbuch nur schwierig zu erhalten sind, oder die vorgeschlagenen Kollokationen nicht typisch sind: notwehrfähig sein (etwas ist notwehrfähig: Rechtsgüter des Staates sind notwehrfähig) = silobran je možen pri..., Vorstrafen aufweisen = biti kaznovan zaradi istovrstnega kaznivega dejanja, den Mietzins mindern ${ }^{8}=$ znižati najemnino, Kündigungsschutz (und Räumungsschutz) genießen = uživati pravno varstvo zoper odpoved (in izselitev iz stanovanja), für eigene/fremde Rechnung handeln' = nastopati za/na svoj/tuj račun, unter Verwendung

\footnotetext{
7 Bei den verbalen Kollokationen (substantivischer Terminus als Basis + Verb als Kollokator oder Adjektiv + Verb) erweist sich die Wahi solcher Verben oft als besonders schwierig, die für einen fachsprachlichen Kontext angemessen sind. Weniger allerdings dann, wenn „es sich um rein fachsprachliche Verben handelt, die auch vom Nichtfachmann leicht als solche erkannt werden ...(Picht 1988, 187). Problematischer wird es dann, wenn scheinbar „harmlose gemeinsprachliche Verben" eingesetzt werden müssen (vgl. die Beispiele).

8 In Apovnik/Karničar (1989: 202) findet sich unter Minderung nur $\sim$ des Kaufpreises = znižanje kupnine.

9 In Apovnik/Karničar (1989: 242) kommt unter Rechnung nur die Wortverbindung aufffür eigene $\sim$ und für eines anderen vor, d.h. ohne das typische Verb handeln.
} 
einer Waffe $=z$ uporabo orožja, sich rechtswidrig zueignen $=$ protipravno prisvojiti si, den Kaufgegenstand besichtigen = pregledati predmet pogodbe, zu Wohnzwecken vermieten $=$ dati $v$ najem za bivanje/kot bivalne prostore, Anspruch auf etw. haben = biti upravičen do česa, in Kraft treten ${ }^{10}=$ stopiti $v$ veljavo. Als Übersetzer/in ist man bei der Suche nach typischen Kollokationen in der Zielsprache auf andere Quellen angewiesen - etwa auf relevante Abschnitte eines Handbuches oder auf Paralleltexte, was aber zeitaufwendig sein kann.

Wie schon gesagt, stellt das Übersetzen von Kollokationen eine große Herausforderung dar. Wichtig dabei ist es daher, „dass den Übersetzern zumindest gute lexikographische und terminographische Hilfsmittel beiseite stehen. Dies gilt vor allem für den Bereich der Fachsprachen, da hier das Kollokationswissen auch für die Herübersetzung in der Muttersprache nicht vorausgesetzt werden kann" (Caro Cedillo 2004: 34). Der Bedarf an Kollokationsangaben in Fachwörterbüchern ist groß, insbesondere in solchen Fachwörterbüchern, deren intendierte Adressaten auch Laien ${ }^{11}$ sind. Von Vorteil wären Fachwörterbücher a) mit einer eindeutigen Makrostruktur, wo mehr oder weniger klar zwischen Termini und Nicht-Temini (d. h. Kollokationen) unterschieden wird, b) mit einer präzisen Mikrostruktur, wo die Kollokationen im Rahmen des Wörterbuchartikels immer einen bestimmten Platz haben und konsequent entweder unter der Basis bzw. unter dem Kollokator vorkommen und schließlich c) Fachwörterbücher, die reich an Kollokationen sind und als solche den Benutzern bei der Produktion in der Fachsprache zuverlässig beiseite stehen.

\section{Literatur}

APOVNIK, Pavel / Ludvik Karničar (1989) Wörterbuch der Rechts- und Wirtschaftssprache.

BERGENHOLTZ, Henning/Sven TARP (1994) Mehrworttermini und Kollokationen in Fachwörterbüchern. In: Burkhard Schaeder/ Henning Bergenholtz (Hrsg.), Fachlexikographie. Fachwissen und seine Repräsentation in Wörterbüchern. Tübingen: Narr, 385-419. (Forum für Fachsprachen-Forschung, 23).

BURGER, Harald (2003) Phraseologie. Eine Einführung am Beispiel des Deutschen. 2. überarbeitete Aufl. Berlin: Erich Schmidt Verlag.

CARO CEDILLO, Anna (2004) Fachsprachliche Kollokationen. Ein übersetzungsorientiertes Datenbankmodell Deutsch-Spanisch. Tübingen: Narr. (Forum für Fachsprachen-Forschung, 63).

COULMAS, Florian (1981) Routine im Gespräch: zur pragmatischen Fundierung der Idiomatik. Wiesbaden: Akad. Verl.-Ges.-Athenaion (Linguistische Forschungen, 29).

CREIFELDS, Carl (1996) Rechtswörterbuch. 13. neubearbeitete Auflage. München: Beck.

FIRTH, John Rupert (1957) Modes of Meaning. In: Papers in Linguistics 1934.1951. London: Oxford University Press.

FLEISCHER, Wolfgang (1997) Phraseologie der deutschen Gegenwartssprache. 2. durchgesehene und ergänzte Aufl. Tübingen: Niemeyer.

HAUSMANN, Franz Josef (1985) Kollokationen im deutschen Wörterbuch. Ein Beitrag zur Theorie des lexikographischen Beispiels. In: Henning Bergenholtz / Joachim Mugdan (Hrsg.), Lexikographie und Grammatik. Akten des Essener Kolloquiums zur Grammatik im Wörterbuch 28.-30. 6. 1984. Tübingen: Niemeyer.

HAUSMANN, Franz Josef (2003) Kollokationen in der Fachsprache: Schwerpunkt Französisch. In: Udo O. H. Jung / Angelina Kolesnikova (Hrsg.), Fachsprachen und Hochschule. 2003 Fankfurt/a. Main: Peter Lang, 83-92.

\footnotetext{
${ }^{10}$ In Apovnik/Karničar (1989: 177) wird in Kraft treten als začeti veljati übersetzt, was keine typische Kollokation ist.

${ }^{11}$ Laut Schaeder $(1994,70)$ sind Laien fachlich interessierte Laien (dazu zählen auch Übersetzer) oder angehende Fachleute (Lerner) des betreffenden Faches.
} 
IRSULA PEÑA, Jesus (1994) Substantiv-Verb-Kollokationen: kontrastive Untersuchungen Deutsch-Spanisch. Frankfurt/Main et al.: Lang.

KJÆR, Anne Lise (1990) Phraseology research, State-of-the-art: Methods of describing word combinations in language for specific purposes. IITF Journal 1: 1-2, 3-20.

KOZIOL, Helmut / Rudolf WELSER (1995) Grundriß des bürgerlichen Rechts. Band 1: Allgemeiner Teil und Schuldrecht. 10. Aufl. Wien: Manzsche Verlags- und Universitätsbuchhandlung.

LEHR, Andrea (1993) Kollokationsanalysen: von der Kollokationstheorie des Kontextualismus zu einem computerunterstützten Verfahren. Zeitschrift für germanistische Linguistik, 2-19.

PICHT, Heribert (1988) Fachsprachliche Phraseologie. In: Reiner Arntz (Hg.), Textlinguistik und Fachsprache. ALLA-Symposion Hildesheim, 13. - 16. April 1987. Hildesheim, Zürich, New York: Georg Olms Verlag, 187-196.

SCHAEDER, Burkhard (1994) Das Fachwörterbuch als Darstellungsform fachlicher Wissensbestände. In: Burkhard Schaeder / Henning Bergenholtz (Hrsg.), Fachlexikographie. Fachwissen und seine Repräsentation in Wörterbüchern. Tübingen: Narr, 69-102. (Forum für Fachsprachen-Forschung, 23).

SCHNEIDER, Franz (1998) Studien zur kontextuellen Fachlexikographie: Das deutsch-französische Wörterbuch der Rechnungslegung. Tübingen: Niemeyer.

VIEHWEGER, Dieter (1982) Semantiktheorie und praktische Lexikographie. Zeitschrift für germanistik 1/82, 143-155.

VIEHWEGER, Dieter (1998) Kollokationen im Lexikon und deren Darstellung im Wörterbuch. In: R. Hessky / E. Knipf (Hrsg.), Lexikologie I. Budapest: Holnap Kiado. 187-203.

WIESMANN, Eva (2004) Rechtsübersetzung und Hilfsmittel zur Translation. Tübingen: Narr. (Forum für Fachsprachen-Forschung, 65).

\section{Povzetek}

\section{KOLOKACIJE S TEŽIŠČEM NA NEMŠKO-SLOVENSKEM STROKOVNEM JEZIKU}

Strokovni jezik ima dve dimenziji: posamezno besedo, termin, ki je osnovna enota terminologije, in kontekstualizacijo termina v obliki kolokacij. Iskanje ekvivalentnih terminov je pri pravnih besedilih neredko zahtevna naloga. Kdor prevaja pravna besedila, namreč nima opraviti le $s$ strokovnim področjem $v$ dveh različnih jezikih, temveč tudi $z$ dvema pravnima redoma. Zelo zahtevna je v pravu ne le izbira primernih terminov, ampak tudi raba ustreznih glagolov, pridevnikov in predlogov, ki se s termini povezujejo, npr: einen Vertrag schließen/aufheben $=$ skleniti/razveljaviti pogodbo, unter Verwendung einer Waffe $=z$ uporabo orožja, das Gesetz verabschieden = sprejeti zakon itn. Take besedne zveze tudi izkušene prevajalce postavljajo pred težavne naloge, saj oblikujejo prevodne celote in jih zato ni mogoče prevajati segmentirano oz. na podlagi zgolj besednih ustreznic. Kolokacije $\mathrm{v}$ strokovnem jeziku povzročajo težave ne le pri prevajanju $v$ tuji, pač pa tudi v materni jezik. Vzrok je na eni strani v tem, da večinoma vsak strokovni jezik vsebuje besedne zveze, ki v splošnem jeziku ne obstajajo. Poleg tega pa mnogih tovrstnih besednih zvez ni najti v različnih priročnikih kot so leksikoni, glosarji, slovarji ipd. V pričujočem članku se v poglavitnem posvečam kolokacijam in neogibnosti njihovega vključevanja $\mathrm{v}$ strokovne slovarje. 